\title{
Spectral characterizations of dumbbell graphs
}

\author{
Jianfeng Wang* \\ Department of Mathematics \\ Qinghai Normal University \\ Xining, Qinghai 810008, P.R. China \\ jfwang4@yahoo.com.cn \\ Qiongxiang Huang \\ College of Mathematics and System Science \\ Xinjiang University \\ Urumqi 830046, P.R. China \\ huangqx@xju.edu.cn
}

\author{
Francesco Belardo ${ }^{\dagger}$ \\ Department of Mathematics \\ University of Messina \\ Sant'Agata 98166, Messina, Italy \\ fbelardo@gmail.com \\ Enzo M. Li Marzi \\ Department of Mathematics \\ University of Messina \\ Sant'Agata 98166, Messina, Italy \\ emlimarzi@gmail.com
}

Submitted: Jul 13, 2009; Accepted: Mar 4, 2010; Published: Mar 15, 2010

Mathematics Subject Classifications: 05C50

\begin{abstract}
A dumbbell graph, denoted by $D_{a, b, c}$, is a bicyclic graph consisting of two vertexdisjoint cycles $C_{a}, C_{b}$ and a path $P_{c+3}(c \geqslant-1)$ joining them having only its end-vertices in common with the two cycles. In this paper, we study the spectral characterization w.r.t. the adjacency spectrum of $D_{a, b, 0}$ (without cycles $C_{4}$ ) with $\operatorname{gcd}(a, b) \geqslant 3$, and we complete the research started in [J.F. Wang et al., A note on the spectral characterization of dumbbell graphs, Linear Algebra Appl. 431 (2009) 1707-1714]. In particular we show that $D_{a, b, 0}$ with $3 \leqslant \operatorname{gcd}(a, b)<a$ or $\operatorname{gcd}(a, b)=a$ and $b \neq 3 a$ is determined by the spectrum. For $b=3 a$, we determine the unique graph cospectral with $D_{a, 3 a, 0}$. Furthermore we give the spectral characterization w.r.t. the signless Laplacian spectrum of all dumbbell graphs.
\end{abstract}

\section{Introduction}

Let $G=(V(G), E(G))$ be a graph with order $|V(G)|=n(G)=n$ and $\operatorname{size}|E(G)|=$ $m(G)=m$. Let $A(G)$ be the $(0,1)$-adjacency matrix of $G$ and $d_{G}(v)=d(v)$ the degree of the vertex $v$. The polynomial $\phi(G, \lambda)=\operatorname{det}(\lambda I-A(G))$ or simply $\phi(G)$, where $I$ is the

*Research supported by the NSFC (No. 10761008 and No. 10961023) and the XGEDU 2009 S20.

${ }^{\dagger}$ Research supported by the INdAM (Italy). 
identity matrix, is defined as the characteristic polynomial of $G$, which can be written as $\phi(G)=\lambda^{n}+a_{1}(G) \lambda^{n-1}+a_{2}(G) \lambda^{n-2}+\cdots+a_{n}(G)$. Since $A(G)$ is real and symmetric, its eigenvalues are all real numbers. Assume that $\lambda_{1}(G) \geqslant \lambda_{2}(G) \geqslant \cdots \geqslant \lambda_{n}(G)$ are the adjacency eigenvalues of the graph $G$. The adjacency spectrum of $G$, denoted by $\operatorname{Spec}(G)$, is the multiset of its adjancency eigenvalues.

Together with the adjacency spectrum, shortly denoted by A-spectrum, we will consider the $Q$-spectrum, defined similarly but with respect to the signless Laplacian matrix $Q(G)=A(G)+D(G)$, where $D(G)$ is the diagonal matrix of vertex degrees (of $G$ ). The same applies for eigenvalues, characteristic polynomial, and the corresponding notation differs by a prefix ( $A$ - or $Q$-, respectively). The characteristic polynomials of the matrices $A(G)$ and $Q(G)$ will be denoted by $\phi(G, \lambda)$ and $\varphi(G, \lambda)$, respectively; we will omit the variable if it is clear from the context. According to [3, 4, 5], all these approaches (with different matrices $M$ ) fit into the so called $M$-theory of graph spectra, and moreover there are some very helpful analogies between them.

In this paper, let $M$ be the adjacency matrix $A$ or the signless Laplacian matrix $Q$. Two graphs are said to be $M$-cospectral (or that they are $M$-cospectral mates) if they have equal $M$-spectrum, i.e. equal $M$-characteristic polynomial. A graph is said to be determined by its $M$-spectrum, or shortly $D M S$, if there is no other non-isomorphic graph with the same $M$-spectrum. Numerous examples of $M$-cospectral but non-isomorphic graphs, known as $M$-PINGS, are reported in the literature (see Chapter 6 in [2] for example). On the other hand, only a few graphs with very special structure have been proved to be determined by their $M$-spectra. For the background and some known results about this problem and related topics, we refer the readers to the excellent surveys $[6,7]$ and the references therein.

As usual, let $C_{n}$ and $P_{n}$ be, respectively, the cycle, and the path of order $n$. For two graphs $G$ and $H, G \cup H$ denotes the disjoint union of $G$ and $H$. Let $T_{a, b, c}$ denote the tree with exactly one vertex $v$ having maximum degree 3 such that $T_{a, b, c}-v=P_{a} \cup P_{b} \cup P_{c}$. The lollipop graph, denoted by $L_{g, p}$ (note, in [10] $L_{g, p}$ is denoted by $H_{g+p, g}$ ), is obtained by appending a cycle $C_{g}$ to a pendant vertex of a path $P_{p+1}$. The $\theta$-graph, denoted by $\theta_{a_{1}, b_{1}, c_{1}}$ $\left(a_{1} \leqslant b_{1} \leqslant c_{1}\right.$ and $\left.\left(a_{1}, b_{1}\right) \neq(0,0)\right)$, is a graph consisting of two given vertices joined by three vertex disjoint paths whose orders are $a_{1}, b_{1}$ and $c_{1}$, respectively. The dumbbell graph $D_{a, b, c}$ consists of two vertex-disjoint cycles $C_{a}, C_{b}$ and a path $P_{c+3}(c \geqslant-1)$ joining them having only its end-vertices in common with the cycles (see Fig. 1). A graph $G$ is said to be almost regular if $\left|d\left(v_{i}\right)-d\left(v_{j}\right)\right| \leqslant 1$ for any $v_{i}, v_{j} \in V(G)$. Clearly, there are two types of such graphs: one is the regular graph and the other one is called $(r, r+1)$-almost regular graph, i.e., its vertex set can be partitioned into two subsets $V_{1}$ and $V_{2}$ such that $d\left(v_{i}\right)=r$ for $v_{i} \in V_{1}$ and $d\left(v_{j}\right)=r+1$ for $v_{j} \in V_{2}$. Note, there are exactly two kinds of $(2,3)$-almost regular graphs such that $m=n+1$, and such graphs are the dumbbell graphs or the $\theta$-graphs with eventually cycles as connected components.

In [10] and [1], the authors shown that all lollipop graphs are DAS. In [11] the authors shown that all $\theta$-graphs with no unique cycle $C_{4}$ are DAS. In [12], we investigated the $A$-spectral characterization of dumbbell graphs without cycles $C_{4}$ and we left the special case $D_{a, b, 0}$ with $\delta=\operatorname{gcd}(a, b) \geqslant 3$. 
In this paper, we will show that $D_{a, b, 0}$ with $\delta=\operatorname{gcd}(a, b) \geqslant 3$ is DAS if and only if $\delta \neq a$ or $\delta=a$ and $b \neq 3 a$. For $b=3 a(a \neq 4)$ we determine the unique graph $A$-cospectral with $D_{a, 3 a, 0}$, that is $\theta_{1, a-1,2 a-1} \cup C_{a}$.

Furthermore we deduce from our main result the $Q$-spectral characterization of dumbbell graphs. In particular we prove that all dumbbell graphs $D_{a, b, c} \neq D_{a, 3 a,-1}$ are DQS, while $D_{a, 3 a,-1}$ is $Q$-cospectral just with $\theta_{0, a-1,2 a-1} \cup C_{a}$.

The paper is organized as follows. In Section 2 we give a few basic results that will be used later. In Section 3 we restrict the structure of tentative $A$-cospectral mates with $D_{a, b, 0}$. In Section 4 we give the $A$-spectral characterization of $D_{a, b, 0}$ and we give the general result on the $A$-spectral characterization of $D_{a, b, c}$ without cycle $C_{4}$ as subgraph. Finally in Section 5, we give the $Q$-spectral characterization of $D_{a, b, c}$. Note that in order to keep the notation easier to read, we will omit the prefix $A$ - in Sections 2, 3 and 4 since the latter sections are concerning just with the $A$-theory of graph spectra, while we again make use of the prefixes $A$ - and $Q$ - in Section 5 .
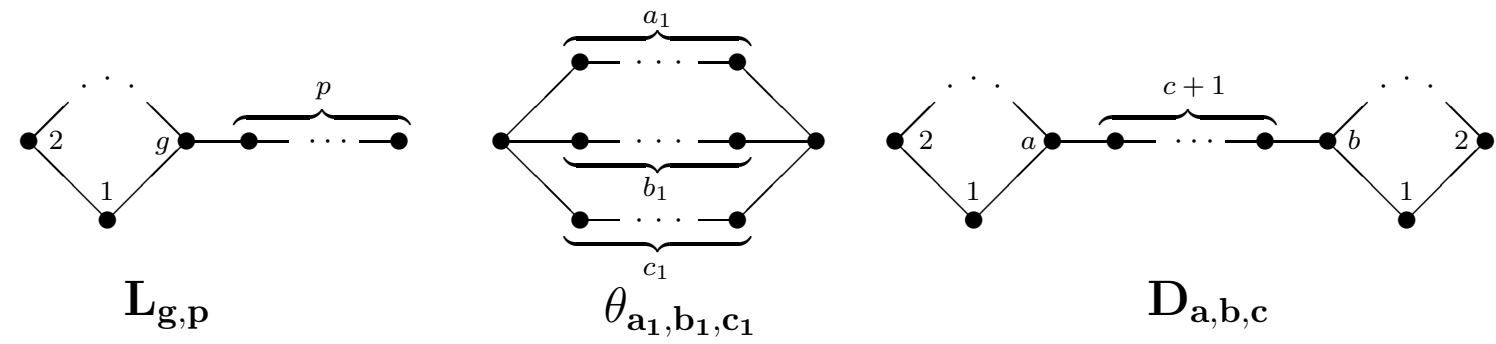

Fig. 1: The graphs $L_{g, p}, \theta_{a_{1}, b_{1}, c_{1}}$ and $D_{a, b, c}$.

Remark 1. Due to the symmetry, let $0 \leqslant a_{1} \leqslant b_{1} \leqslant c_{1}$ in the graph $\theta_{a_{1}, b_{1}, c_{1}}$ and $3 \leqslant a \leqslant b$ and $c \geqslant-1$ in the graph $D_{a, b, c}$.

\section{Basic results}

Some useful established results about the $(A$-)spectrum are presented in this section, which will play an important role throughout this paper. Recall that the prefix $A$ - is omitted in this section.

Lemma 2.1 (Interlacing Theorem). Let the eigenvalues of graphs $G$ and $G-v$ be, respectively, $\lambda_{1} \geqslant \lambda_{2} \geqslant \cdots \geqslant \lambda_{n}$ and $\mu_{1} \geqslant \mu_{2} \geqslant \cdots \geqslant \mu_{n-1}$, then $\lambda_{1} \geqslant \mu_{1} \geqslant \lambda_{2} \geqslant \mu_{2} \geqslant$ $\cdots \geqslant \mu_{n-1} \geqslant \lambda_{n}$.

Lemma 2.2 (Schwenk's formulas). [2] Let $G$ be a (simple) graph. Denote by $\mathscr{C}(v)(\mathscr{C}(e))$ the set of all cycles in $G$ containing a vertex $v$ (resp. an edge $e=u v$ ). Then we have:

(i) $\phi(G, x)=x \phi(G-v, x)-\sum_{w \sim v} \phi(G-v-w, x)-2 \sum_{C \in \mathscr{C}(v)} \phi(G-V(C), x)$

(ii) $\phi(G, x)=\phi(G-e, x)-\phi(G-v-u, x)-2 \sum_{C \in \mathscr{C}(e)} \phi(G-V(C), x)$. 
We assume that $\phi(G, x)=1$ if $G$ is the empty graph (i.e. with no vertices).

Lemma 2.3. [2] Let $C_{n}$ and $P_{n}$ be the cycle and the path on $n$ vertices, respectively. Then

(i) $\phi\left(C_{n}\right)=\prod_{j=1}^{n}\left(\lambda-2 \cos \frac{2 \pi j}{n}\right)$ and $\lambda_{1}\left(C_{n}\right)=2$,

(ii) $\phi\left(P_{n}\right)=\prod_{j=1}^{n}\left(\lambda-2 \cos \frac{\pi j}{n+1}\right)$ and $\lambda_{1}\left(P_{n}\right)<2$.

Lemma 2.4. [6] Let $G$ and $H$ be two graphs with the same spectrum w.r.t. A or $Q$. Then

(i) $n(G)=n(H)$;

(ii) $m(G)=m(H)$.

Lemma 2.5. [8] $\phi\left(P_{n}, 2\right)=n+1$ and $\phi\left(T_{a, b, c}, 2\right)=a+b+c+2-a b c$.

From the above lemma, in [12] we got the following result.

Lemma 2.6. $2 \in \operatorname{Spec}\left(D_{a, b, c}\right)$ if and only if $c=0$. Moveover, the multiplicity of 2's is one.

The following result describes the structure of tentative cospectral mates of almost regular graphs non containing cycles $C_{4}$ as subgraphs.

Theorem 2.7. [12] Let two graphs $H$ and $G$ such that $\operatorname{Spec}(H)=\operatorname{Spec}(G)$, where $G$ contains no the cycle $C_{4}$ as its subgraph. If $G$ is a $(r, r+1)$-almost regular graph, then

(i) $H$ contains no the cycle $C_{4}$ as its subgraph;

(ii) $H$ is a $(r, r+1)$-almost regular graph with the same degree sequence as $G$.

\section{Preliminary results}

In this section we will restrict the structure of $H$, the tentative $(A$-) cospectral mate of $D_{a, b, 0}$. Recall that the prefix $A$ - is omitted in this section. Note that from Theorem 2.7, $H$ can be a dumbbell graph, a $\theta$-graph, a disjoint union of a dumbbell graph and cycles, a disjoint union of a $\theta$-graph and cycles. Since $D_{a, b, 0}$ has 2 as an eigenvalue of multiplicity 1 (cf. Lemma 2.6) then $H$ contains at most one cycle as connected component. Furthermore, the tentative connected cospectral mates are immediately discarded by the two following lemmas (see, for example, [12]).

Lemma 3.1. [11] There is no $\theta$-graph cospectral with a dumbbell graph.

Lemma 3.2. [12] No two non-isomorphic dumbbell graphs are cospectral.

In [12] we considered the spectral characterization of dumbbell graphs. Our main result reads: 
Theorem 3.3. The graphs $D_{a, b, c}$, without cycles $C_{4}$, with $c \neq 0$ or $c=0$ and $\operatorname{gcd}(a, b) \leqslant 2$ are determined by their adjacency spectrum.

Our aim in this paper is to study the spectral characterization of the remaining cases of Theorem 3.3, i.e. $D_{a, b, 0}$ with $\operatorname{gcd}(a, b) \geqslant 3$. So in the rest of the paper we set $\delta=\operatorname{gcd}(a, b)$ and $\delta \geqslant 3$.

To prove the next lemmas we will rely on the Schwenk's formulas and the Interlacing Theorem. The main idea is the following: if a graph has some eigenvalues of multiplicity greater than 2, then these eigenvalues must appear at least once in all subgraphs obtained by deleting a vertex (from Interlacing Theorem). Hence, we can check the multiplicity of these eigenvalues of vertex deleted subgraphs by substituting them into the characteristic polynomial of the parent graph (by using the Schwenk's formulas). The following lemma characterizes the spectrum of $D_{a, b, 0}($ with $\delta \geqslant 3$ ).

Lemma 3.4. The spectrum of $D_{a, b, 0}$ with $\delta=\operatorname{gcd}(a, b) \geqslant 3$ consists of the eigenvalues of $C_{\delta}$ (except 2 and -2) with multiplicity 3 , the eigenvalues of $C_{a}$ and $C_{b}$ not in $C_{\delta}$ with multiplicity 1 and all the other eigenvalues must strictly interlace the eigenvalues of $C_{a} \cup C_{b}$ and have multiplicity 1 as well.

Proof. If we consider the Interlacing Theorem (Lemma 2.1) applied to the unique cutvertex $u$ of degree 2 in $D_{a, b, 0}$ we get that if $\bar{\lambda}$ is of multiplicity $\geqslant 2$ then $\bar{\lambda} \in \operatorname{Spec}\left(C_{a}\right) \cup$ $\operatorname{Spec}\left(C_{b}\right)$. Consider now the Lemma 2.2(i) applied to $u$. We get:

$$
\phi\left(D_{a, b, 0}\right)=x \phi\left(C_{a}\right) \phi\left(C_{b}\right)-\phi\left(C_{a}\right) \phi\left(P_{b-1}\right)-\phi\left(P_{a-1}\right) \phi\left(C_{b}\right) .
$$

Now take $\bar{\lambda} \in \operatorname{Spec}\left(C_{\delta}\right)$ and $\bar{\lambda} \neq \pm 2$, it is easy to check that such a $\bar{\lambda}$ is 4 times solution of $\phi\left(C_{a}\right) \phi\left(C_{b}\right), 3$ times solution of $\phi\left(C_{a}\right) \phi\left(P_{b-1}\right)$ and 3 times solution of $\phi\left(P_{a-1}\right) \phi\left(C_{b}\right)$. Consequently $\bar{\lambda} \in \operatorname{Spec}\left(C_{\delta}\right)(\lambda \neq \pm 2)$ implies that $\bar{\lambda}$ is of multiplicity 3 for $D_{a, b, 0}$. If $\bar{\lambda}=2$, then 2 is a simple root of (1) (see also Lemma 2.6); note also that $\lambda_{2}\left(D_{a, b, 0}\right)=2$ (by Interlacing Theorem). If $\bar{\lambda}=-2 \in \operatorname{Spec}\left(C_{\delta}\right)$, then -2 is a simple root of (1) as well.

Take now $\bar{\lambda} \in \operatorname{Spec}\left(C_{a}\right) \cup \operatorname{Spec}\left(C_{b}\right) \backslash \operatorname{Spec}\left(C_{\delta}\right)$, note that $\operatorname{Spec}\left(C_{a}\right) \cap \operatorname{Spec}\left(C_{b}\right)=$ $\operatorname{Spec}\left(C_{\delta}\right)$ (see Lemma 2.3(i)). Similarly to above we can say that such a $\bar{\lambda}$ is an eigenvalue of multiplicity 1 for $D_{a, b, 0}$.

Since all multiple eigenvalues of $D_{a, b, 0}$ must come from $\operatorname{Spec}\left(C_{a}\right) \cup \operatorname{Spec}\left(C_{b}\right)$, then, by Interlacing Theorem, all remaining eigenvalues must interlace the eigenvalues of $C_{a} \cup C_{b}$ and be of multiplicity 1 .

This ends the proof.

From the above lemma we know to some extent the spectrum of $D_{a, b, 0}$. If $H$ is a tentative cospectral mate of $D_{a, b, 0}$, then $H$ cannot be connected (by Theorem 2.7 and Lemmas 3.1 and 3.2). Furthermore by Lemma 2.6 (cf. also Lemma 3.4), we know that 2 is simple and the second largest eigenvalue of $D_{a, b, 0}$. The latter implies that $H$ can be of two kinds: a $\theta$-graph with a cycle or a dumbbell graph with a cycle. The eigenvalues of multiplicity 3 of $D_{a, b, 0}$ (recall that they belong to $C_{\delta}$, by Lemma 3.4) will force the latter mentioned cycles to be $C_{\delta}$. This fact will be proved in the following lemmas. 
Lemma 3.5. If $H=D_{a^{\prime}, b^{\prime}, c^{\prime}} \cup C_{p^{\prime}}$ is cospectral with $D_{a, b, 0}$, then $c^{\prime}=-1$ and $p^{\prime}=\delta$.

Proof. Recall that, by Lemma 2.6, 2 is a simple eigenvalue of $D_{a, b, 0}$. Assume that $\phi\left(D_{a, b, 0}\right)=\phi(H)$. Since $H$ contains a cycle then 2 appears already as an eigenvalue and, consequently, $D_{a^{\prime}, b^{\prime}, c^{\prime}}$ cannot have 2 as its eigenvalue. By Lemma 2.6 we get $c^{\prime} \neq 0$.

Assume that $c^{\prime}>0$. Considering Lemma 2.1 applied to the cut-vertex of degree 2 of $D_{a, b, 0}$, we get that $\lambda_{1}\left(D_{a, b, 0}\right)>\lambda_{1}\left(C_{b}\right) \geqslant \lambda_{2}\left(D_{a, b, 0}\right) \geqslant \lambda_{1}\left(C_{a}\right)$. Consider now $H$, it is easy to see, by using the above argument, that its second largest eigenvalue is (strictly) greater than 2 whenever $c^{\prime}>0$, which is a contradiction.

Take now $c^{\prime}=-1$. So $H=D_{a^{\prime}, b^{\prime},-1} \cup C_{p^{\prime}}$. Recall that by Lemma 3.4, we know that the spectrum of $D_{a, b, 0}$ contains the eigenvalues of $C_{\delta}$ (except \pm 2 ) with multiplicity 3 and the remaining eigenvalues are simple. It is easy to see that $p^{\prime}$ divides $\delta$, otherwise $H$ has some eigenvalues of multiplicity at least 2 not appearing in $D_{a, b, 0}$. Assume, for a contradiction, that $p^{\prime}<\delta$. If so, $H \backslash C_{p^{\prime}}=D_{a^{\prime}, b^{\prime},-1}$ has at least an eigenvalue $\bar{\lambda}$ of multiplicity 3 . By Lemma 2.2(ii) applied at the (unique) bridge of $D_{a^{\prime}, b^{\prime},-1}$, we have

$$
\phi\left(D_{a^{\prime}, b^{\prime},-1}\right)=\phi\left(C_{a^{\prime}}\right) \phi\left(C_{b^{\prime}}\right)-\phi\left(P_{a^{\prime}-1}\right) \phi\left(P_{b^{\prime}-1}\right) .
$$

By Lemma 2.1 applied at the vertex of degree 3 in $C_{b^{\prime}}$, we have that $\bar{\lambda} \in \operatorname{Spec}\left(C_{a^{\prime}}\right)$, and by the same lemma applied at the other vertex of degree 3 we have that $\bar{\lambda} \in \operatorname{Spec}\left(C_{b^{\prime}}\right)$. Hence from (2), $\bar{\lambda}$ is exactly of multiplicity 2 in $D_{a^{\prime}, b^{\prime},-1}$, that is a contradiction. So the eigenvalues of $H \backslash C_{p^{\prime}}=D_{a^{\prime}, b^{\prime},-1}$ are simple and, consequently, it must be $\delta=p^{\prime}$.

Lemma 3.6. Let $L_{g, p}$ be a lollipop. If $\bar{\lambda} \in \operatorname{Spec}\left(L_{g, p}\right)$ is of multiplicity greater than 1, then its multiplicity is exactly 2 and $\bar{\lambda} \in \operatorname{Spec}\left(C_{g}\right) \cap \operatorname{Spec}\left(P_{p-1}\right)$.

Proof. Recall that from Lemma 2.3 we have the following facts: if $\lambda \in \operatorname{Spec}\left(C_{n}\right)$ then $\lambda \in \operatorname{Spec}\left(P_{n-1}\right)$; if $\lambda \in \operatorname{Spec}\left(P_{n}\right)$ then $\lambda \notin \operatorname{Spec}\left(P_{n-1}\right)$; if $\lambda \in \operatorname{Spec}\left(P_{n}\right)$ then $\lambda$ is of multiplicity 1.

Assume that $\bar{\lambda}$ is of multiplicity at least 2 for $L_{g, p}$. By the Interlacing Theorem applied at the vertex of degree 2 in the path adjacent to the vertex of degree $3, \bar{\lambda}$ must be an eigenvalue of $C_{g}$ or of $P_{p-1}$. Consider now the Schwenk formula for edges (Lemma 2.2(ii)) at the bridge between the path and the cycle in $L_{g, p}$. We have

$$
\phi\left(L_{g, p}\right)=\phi\left(C_{g}\right) \phi\left(P_{p}\right)-\phi\left(P_{g-1}\right) \phi\left(P_{p-1}\right) .
$$

It easy to see that if $\bar{\lambda}$ is an eigenvalue of $C_{g}$ in (3) then such an eigenvalue is an eigenvalue of $P_{p-1}$ (recall that $\bar{\lambda}$ is of multiplicity at least 2 ) as well, while if $\bar{\lambda}$ is an eigenvalue of $P_{p-1}$ then (3) holds if and only if such an eigenvalue belongs to $\operatorname{Spec}\left(C_{g}\right)$ as well. So we can conclude that $\bar{\lambda} \in \operatorname{Spec}\left(C_{g}\right) \cap \operatorname{Spec}\left(P_{p-1}\right)$. Finally, it is easy to observe that such a $\bar{\lambda} \in \operatorname{Spec}\left(C_{g}\right) \cap \operatorname{Spec}\left(P_{p-1}\right)$ is a solution of $(3)$ exactly twice.

This ends the proof.

Lemma 3.7. Let $\bar{\lambda}$ be an eigenvalue of multiplicity at least 3 for $\theta_{a_{1}, b_{1}, c_{1}}$. Then the multiplicity of $\bar{\lambda}$ is exactly $3, a_{1}, b_{1}$ and $c_{1}$ are odd integers and $\bar{\lambda}=0$. 
Proof. Let $\bar{\lambda}$ be an eigenvalue of multiplicity at least 3 for $\theta_{a_{1}, b_{1}, c_{1}}$, then, by the Interlacing theorem (Lemma 2.1), $\bar{\lambda}$ is an eigenvalue of multiplicity (at least) 2 in all vertex deleted subgraphs of $\theta_{a_{1}, b_{1}, c_{1}}$. Assume that the multiplicity of $\bar{\lambda}$ is strictly greater than 3 , then $\bar{\lambda}$ is of multiplicity at least 3 in all vertex deleted subgraphs, including the lollipop graphs and cycles, but by Lemmas 3.6 and 2.3 we have that this is impossible. So in the rest we assume that $\bar{\lambda}$ is of multiplicity exactly 3 .

Assume first that $a_{1}>2$ and consider the three lollipops coming from $\theta_{a_{1}, b_{1}, c_{1}}$ by deleting a vertex. It is easy to see that these three lollipops are indeed $L_{a_{1}+b_{1}+2, c_{1}-1}$, $L_{a_{1}+c_{1}+2, b_{1}-1}$ and $L_{b_{1}+c_{1}+2, a_{1}-1}$.

From Lemma 3.6, if $L_{g, m-1}$ has an eigenvalue of multiplicity 2 then such an eigenvalue belongs to $\operatorname{Spec}\left(C_{g}\right) \cap \operatorname{Spec}\left(P_{m-2}\right)$, and in particular $\bar{\lambda} \in \operatorname{Spec}\left(P_{m-2}\right)$. If we look to $\bar{\lambda}$ as an eigenvalue of multiplicity 3 in $\theta_{a_{1}, b_{1}, c_{1}}$ we get the following condition:

$$
\bar{\lambda} \in \operatorname{Spec}\left(P_{a_{1}-2}\right) \cap \operatorname{Spec}\left(P_{b_{1}-2}\right) \cap \operatorname{Spec}\left(P_{c_{1}-2}\right)
$$

Consider now the vertex deleted subgraph of $\theta_{a_{1}, b_{1}, c_{1}}$, i.e. $T_{a_{1}, b_{1}, c_{1}}$. By reasoning in a similar way as above we get that $\bar{\lambda}$ is an eigenvalue of multiplicity 2 of $T_{a_{1}, b_{1}, c_{1}}$ and, consequently, $\bar{\lambda}$ is an eigenvalue of any vertex deleted subgraph of $T_{a_{1}, b_{1}, c_{1}}$, including $P_{a_{1}} \cup P_{b_{1}} \cup P_{c_{1}}$. So we get:

$$
\bar{\lambda} \in \operatorname{Spec}\left(P_{a_{1}}\right) \cup \operatorname{Spec}\left(P_{b_{1}}\right) \cup \operatorname{Spec}\left(P_{c_{1}}\right) .
$$

By combining (4) and (5), we get that the only possibility is that $a_{1}, b_{1}$ and $c_{1}$ are odd integers and $\bar{\lambda}=0$. In fact, if $\bar{\lambda} \in \operatorname{Spec}\left(P_{a_{1}}\right)$ (if $\bar{\lambda} \in \operatorname{Spec}\left(P_{b_{1}}\right)$ or $\bar{\lambda} \in \operatorname{Spec}\left(P_{c_{1}}\right)$ the proof is analogous) then $\bar{\lambda} \in \operatorname{Spec}\left(P_{a_{1}-2}\right)$ if and only if $a_{1}$ is odd and $\bar{\lambda}=0$, but this implies that $0 \in \operatorname{Spec}\left(P_{b_{1}-2}\right) \cap \operatorname{Spec}\left(P_{c_{1}-2}\right)$ which means that $b_{1}$ and $c_{1}$ are odd numbers as well.

Assume now that $a_{1}=0$, then $\bar{\lambda}$ cannot be an eigenvalue of multiplicity 2 for $T_{a_{1}, b_{1}, c_{1}}=$ $P_{b_{1}+c_{1}+1}$, so we must consider only the cases $a_{1}=1$ and $a_{1}=2$. Suppose first that $a_{1}=1$. If $\bar{\lambda} \in \operatorname{Spec}\left(P_{a_{1}}\right)$ then $\bar{\lambda}=0$ and $b_{1}, c_{1}$ are odd integers. Otherwise, if $\bar{\lambda} \in$ $\operatorname{Spec}\left(P_{b_{1}}\right) \cup \operatorname{Spec}\left(P_{c_{1}}\right)$, then we can procede as above. Finally, let us consider the case $a_{1}=2$. By applying (3) at this situation we obtain that $L_{b_{1}+c_{1}+2,1}$ cannot have any eigenvalue $\bar{\lambda}$ of multiplicity 2 .

Lemma 3.8. If $H=\theta_{a_{1}, b_{1}, c_{1}} \cup C_{d_{1}}$ is cospectral with $D_{a, b, 0}$, then $d_{1}=\delta$.

Proof. Since $C_{d_{1}}$ contributes to $\operatorname{Spec}(H)$ with eigenvalues of multiplicity 2, we have that $d_{1}$ divides $\delta$. Note that if $\delta \leqslant 5$ then $d_{1}=\delta$ (otherwise $d_{1}=1$ or $d_{1}=2$, impossible). If $\delta=6$ and $d_{1}=3$, then $H=\theta_{a_{1}, b_{1}, c_{1}} \cup C_{3}$ has 1 as an eigenvalue of multiplicity 3, with $1 \in \operatorname{Spec}\left(\theta_{a_{1}, b_{1}, c_{1}}\right)$, impossible by Lemma 3.7. So let $\delta \geqslant 7$, if so any $\lambda \in$ $\operatorname{Spec}(H) \cap \operatorname{Spec}\left(C_{\delta}\right) \backslash\{ \pm 2\}$ is of multiplicity three and all other eigenvalues of $H$ are simple. If $d_{1}<\delta$, then $\theta_{a_{1}, b_{1}, c_{1}}$ must have at least two eigenvalues of multiplicity 3 . The latter fact is a contradiction, since from Lemma 3.7 we have that at most one eigenvalue (i.e. 0 ) can be of multiplicity 3 in $\theta_{a_{1}, b_{1}, c_{1}}$. This means that all eigenvalues of multiplicity 3 in $H$ must be eigenvalues of $C_{d_{1}}$, which implies $d_{1}=\delta$. 


\section{$4 \quad A$-spectral characterization of dumbbell graphs}

Recall that the prefix $A$ - is omitted in this section. By Lemmas 3.5 and 3.8 and we have that a tentative $\left(A\right.$-)cospectral mate with $D_{a, b, 0}$ reduces to $H=D_{a^{\prime}, b^{\prime},-1} \cup C_{\delta}$ or $H=\theta_{a_{1}, b_{1}, c_{1}} \cup C_{\delta}$. Furthermore $\phi\left(C_{\delta}\right)$ divides both $\phi\left(D_{a, b, 0}\right)$ and $\phi(H)$, then we can just compare $\phi\left(D_{a, b, 0}\right) / \phi\left(C_{\delta}\right)$ with $\phi\left(D_{a^{\prime}, b^{\prime},-1}\right)$ and $\phi\left(\theta_{a_{1}, b_{1}, c_{1}}\right)$. To make such comparisons, we will follow the idea of Ramezani et al. (see [11]), that is to express the latter mentioned polynomials through the characteristic polynomials of paths. Let us pose $a=\delta \bar{a}$ and $b=\delta \bar{b}$.

By Lemma 2.2, we obtain

$$
\begin{gathered}
\phi\left(C_{a}\right)=\phi\left(P_{a}\right)-\phi\left(P_{a-2}\right)-2 ; \\
\frac{\phi\left(D_{\delta \bar{a}, \delta \bar{b}, 0}\right)}{\phi\left(C_{\delta}\right)}=\lambda \frac{\phi\left(C_{\delta \bar{a}}\right)}{\phi\left(C_{\delta}\right)} \phi\left(C_{\delta \bar{b}}\right)-\frac{\phi\left(C_{\delta \bar{a}}\right)}{\phi\left(C_{\delta}\right)} \phi\left(P_{\delta \bar{b}-1}\right)-\frac{\phi\left(C_{\delta \bar{b}}\right)}{\phi\left(C_{\delta}\right)} \phi\left(P_{\delta \bar{a}-1}\right) ; \\
\phi\left(D_{a^{\prime}, b^{\prime},-1}\right)=\phi\left(C_{a^{\prime}}\right) \phi\left(C_{b^{\prime}}\right)-\phi\left(P_{a^{\prime}-1}\right) \phi\left(P_{b^{\prime}-1}\right) ; \\
\phi\left(\theta_{a_{1}, b_{1}, c_{1}}\right)=\lambda^{2} \phi\left(P_{a_{1}}\right) \phi\left(P_{b_{1}}\right) \phi\left(P_{c_{1}}\right)-2 \lambda\left(\phi\left(P_{a_{1}-1}\right) \phi\left(P_{b_{1}}\right) \phi\left(P_{c_{1}}\right)+\phi\left(P_{a_{1}}\right) \phi\left(P_{b_{1}-1}\right) \phi\left(P_{c_{1}}\right)\right. \\
\left.+\phi\left(P_{a_{1}}\right) \phi\left(P_{b_{1}}\right) \phi\left(P_{c_{1}-1}\right)\right)+2\left(\phi\left(P_{a_{1}-1}\right) \phi\left(P_{b_{1}-1}\right) \phi\left(P_{c_{1}}\right)+\phi\left(P_{a_{1}-1}\right) \phi\left(P_{b_{1}}\right) \phi\left(P_{c_{1}-1}\right)\right. \\
\left.+\phi\left(P_{a_{1}}\right) \phi\left(P_{b_{1}-1}\right) \phi\left(P_{c_{1}-1}\right)\right)+\phi\left(P_{a_{1}-2}\right) \phi\left(P_{b_{1}}\right) \phi\left(P_{c_{1}}\right)+\phi\left(P_{a_{1}}\right) \phi\left(P_{b_{1}-2}\right) \phi\left(P_{c_{1}}\right) \\
+\phi\left(P_{a_{1}}\right) \phi\left(P_{b_{1}}\right) \phi\left(P_{c_{1}-2}\right)-2\left(\phi\left(P_{a_{1}}\right)+\phi\left(P_{b_{1}}\right)+\phi\left(P_{c_{1}}\right)\right) .
\end{gathered}
$$

From $\phi\left(P_{m}\right)=\lambda \phi\left(P_{m-1}\right)-\phi\left(P_{m-2}\right)$, we get, by solving the latter recurrence equation (see [11]), that for $m \geqslant-2$,

$$
\phi\left(P_{m}\right)=\frac{x^{2 m+2}-1}{x^{m+2}-x^{m}}
$$

where $x$ satisfies $x^{2}-\lambda x+1=0$. So we can express the above characteristic polynomials in terms of $x$. Note also that $n\left(\theta_{a_{1}, b_{1}, c_{1}}\right)=n\left(D_{a, b, 0}\right)-n\left(C_{\delta}\right)=n\left(D_{a^{\prime}, b^{\prime},-1}\right)=a+b+1-\delta$. After some computations, we have (we used Derive to make such computations):

$$
\begin{gathered}
\phi\left(C_{a}\right)=x^{a}+x^{-a}-2 \\
D_{1}(a, b, 0 ; x)=\left(x^{2}-1\right)^{3} x^{m+2} \frac{\phi\left(D_{a, b, 0}\right)}{\phi\left(C_{\delta}\right)},
\end{gathered}
$$

where $m=a+b-1-\delta$ and

$$
\begin{aligned}
D_{1}(a, b, 0 ; x) & =\left(x^{2}-1\right)^{2}\left(x^{\delta \bar{a}}-1\right)\left(x^{\delta \bar{b}}-1\right)\left[\left(x^{\delta \bar{a}}\left(x^{\delta \bar{b}}\left(x^{4}-2 x^{2}-1\right)-x^{4}+1\right)+x^{\delta \bar{b}}\left(1-x^{4}\right)\right.\right. \\
& \left.\left.+x^{4}+2 x^{2}-1\right)\right]\left(x^{\delta}-1\right)^{-2} .
\end{aligned}
$$


Note that,

$$
\frac{x^{\delta t}-1}{x^{\delta}-1}=\sum_{i=0}^{t-1} x^{i \delta}
$$

Then, if $\bar{a}=1$ (so $\delta=\delta \bar{a}=a$ and $b=\delta \bar{b}=k a$, for some integer $k$ ), $D_{1}(a, k a, 0 ; x)$ becomes

$$
\left(x^{2}-1\right)^{2}\left[\sum_{i=0}^{k-1} x^{i a}\right]\left[x^{a}\left(x^{k a}\left(x^{4}-2 x^{2}-1\right)-x^{4}+1\right)+x^{k a}\left(1-x^{4}\right)+x^{4}+2 x^{2}-1\right],
$$

specially if $k=1$ (so $b=a$ ) (7) reduces to

$$
x^{2(a+4)}-4 x^{2(a+3)}+4 x^{2(a+2)}-x^{2 a}-2 x^{a+8}+4 x^{a+6}-4 x^{a+2}+2 x^{a}+x^{8}-4 x^{4}+4 x^{2}-1 ;
$$

otherwise if $\bar{a}>1$ (so $\delta<a)$ we have that $D_{1}(\delta \bar{a}, \delta \bar{b}, 0 ; x)$ becomes

$$
\begin{gathered}
\left.\left(x^{2}-1\right)^{2}\left[\sum_{i=0}^{\bar{a}-1} x^{i \delta}\right]\left[\sum_{i=0}^{\bar{b}-1} x^{i \delta}\right]\left[x^{\delta \bar{a}}\left(x^{\delta \bar{b}}\left(x^{4}-2 x^{2}-1\right)-x^{4}+1\right)+x^{\delta \bar{b}}\left(1-x^{4}\right)+x^{4}+2 x^{2}-1\right)\right] \\
D_{2}\left(a^{\prime}, b^{\prime},-1 ; x\right)=\left(x^{2}-1\right)^{3} x^{m+2} \phi\left(D_{a^{\prime}, b^{\prime},-1}\right),
\end{gathered}
$$

where $m=a^{\prime}+b^{\prime}-2=a+b-1-\delta$ and

$$
\begin{aligned}
& D_{2}\left(a^{\prime}, b^{\prime},-1 ; x\right)=x^{2\left(a^{\prime}+b^{\prime}\right)+6}\left(x^{2}-2\right)^{2}-x^{2 a^{\prime}+2 b^{\prime}}-2 x^{2 a^{\prime}+b^{\prime}+6}+6 x^{2 a^{\prime}+b^{\prime}+4}-6 x^{2 a^{\prime}+b^{\prime}+2} \\
&+ 2 x^{2 a^{\prime}+b^{\prime}}+x^{2\left(a^{\prime}+3\right)}-2 x^{2\left(a^{\prime}+2\right)}+2 x^{2\left(a^{\prime}+1\right)}-x^{2 a^{\prime}}-2 x^{a^{\prime}+2 b^{\prime}+6} \\
&+ 6 x^{a^{\prime}+2 b^{\prime}+4}-6 x^{a^{\prime}+2 b^{\prime}+2}+2 x^{a^{\prime}+2 b^{\prime}}+4 x^{a^{\prime}+b^{\prime}+6}-12 x^{a^{\prime}+b^{\prime}+4}+12 x^{a^{\prime}+b^{\prime}+2} \\
&- 4 x^{a^{\prime}+b^{\prime}}-2 x^{a^{\prime}+6}+6 x^{a^{\prime}+4}-6 x^{a^{\prime}+2}+x^{2\left(b^{\prime}+3\right)}-2 x^{2\left(b^{\prime}+2\right)}+2 x^{2\left(b^{\prime}+1\right)} \\
&- x^{2 b^{\prime}}-2 x^{b^{\prime}+6}+6 x^{b^{\prime}+4}-6 x^{b^{\prime}+2}+2 x^{a^{\prime}}+2 x^{b^{\prime}}+x^{6}-4 x^{4}+4 x^{2}-1 . \\
& T\left(a_{1}, b_{1}, c_{1} ; x\right)=\left(x^{2}-1\right)^{3} x^{m+2} \phi\left(\theta_{a_{1}, b_{1}, c_{1}}\right),
\end{aligned}
$$

where $m=a_{1}+b_{1}+c_{1}=a+b-1-\delta$ and

$$
\begin{aligned}
T\left(a_{1}, b_{1}, c_{1} ; x\right) & =x^{2\left(a_{1}+b_{1}+c_{1}\right)+6}\left(x^{2}-2\right)^{2}-4 x^{a_{1}+b_{1}+4}-4 x^{a_{1}+c_{1}+4}-4 x^{b_{1}+c_{1}+4}+2 x^{a_{1}+b_{1}+6} \\
& +2 x^{a_{1}+c_{1}+6}+2 x^{b_{1}+c_{1}+6}-x^{2 a_{1}+2 b_{1}+4}-x^{2 a_{1}+2 c_{1}+4}-x^{2 b_{1}+2 c_{1}+4} \\
& +4 x^{2 a_{1}+b_{1}+c_{1}+6}+4 x^{a_{1}+2 b_{1}+c_{1}+6}+4 x^{a_{1}+b_{1}+2 c_{1}+6}-2 x^{2 a_{1}+b_{1}+c_{1}+4} \\
& -2 x^{a_{1}+2 b_{1}+c_{1}+4}-2 x^{a_{1}+b_{1}+2 c_{1}+4}-2 x^{2 a_{1}+b_{1}+c_{1}+8}-2 x^{a_{1}+2 b_{1}+c_{1}+8} \\
& -2 x^{a_{1}+b_{1}+2 c_{1}+8}+x^{2 a_{1}+6}+x^{2 b_{1}+6}+x^{2 c_{1}+6}+2 x^{a_{1}+b_{1}+2}+2 x^{a_{1}+c_{1}+2} \\
& +2 x^{b_{1}+c_{1}+2}-4 x^{4}+4 x^{2}-1 .
\end{aligned}
$$


If $D_{a, b, 0}$ is cospectral with $D_{a^{\prime}, b^{\prime},-1} \cup C_{\delta}$ or $\theta_{a_{1}, b_{1}, c_{1}} \cup C_{\delta}$, then the polynomials (6) and (10) or (6) and (11) must be the same, respectively. Next, we compare the monomials with lowest exponent of the above polynomials. Unfortunately in some particular cases, from the lowest exponent monomial we cannot distinguish whether the graphs are cospectral or not, so we will compare the rest of the polynomial. Note that $-\left(1-4 x^{2}+4 x^{4}\right)$ is common to all of them, so we will not consider the latter polynomial during the comparisons.

If we look to the lowest exponent monomial (other than $-4 x^{4}+4 x^{2}-1$ ) of the above polynomials, we get for $D_{1}(a, b, 0 ; x)$ :

- $(\delta<a)$ the monomial with minimum exponent is either $-2 x^{\delta}$ if $3 \leqslant \delta<8$, or $-x^{8}$ if $\delta=8$, or $x^{8}$ if $\delta>8$;

- $(\delta=a$ and $k=1)$ the monomial with minimum exponent is either $2 x^{a}$ if $3 \leqslant a<8$, or $3 x^{8}$ if $a=8$, or $x^{8}$ if $a>8$;

- $(\delta=a$ and $k \geqslant 2)$ the monomial with minimum exponent is either $2 x^{a+2}$ if $3 \leqslant a<6$, or $3 x^{8}$ if $a=6$, or $x^{8}$ if $a>6$.

For $D_{2}\left(a^{\prime}, b^{\prime},-1 ; x\right)$, the monomial with minimum exponent can be deduced from $x^{6}+$ $2 x^{a^{\prime}}+2 x^{b^{\prime}}$. Then we have that it is either $x^{6}$ if $a^{\prime}>6$, or $3 x^{6}$ if $a^{\prime}=6<b^{\prime}$, or $5 x^{6}$ if $a^{\prime}=b^{\prime}=6$, or $2 x^{a^{\prime}}$ if $b^{\prime} \neq a^{\prime} \leqslant 5$, or $4 x^{a^{\prime}}$ if $a^{\prime}=b^{\prime} \leqslant 5$.

For $T\left(a_{1}, b_{1}, c_{1} ; x\right)$, we can deduce, similarly to above, the monomial with minimum exponent from $x^{2 a_{1}+6}+x^{2 b_{1}+6}+x^{2 c_{1}+6}+2 x^{a_{1}+b_{1}+2}+2 x^{a_{1}+c_{1}+2}+2 x^{b_{1}+c_{1}+2}$.

Lemma 4.1. $D_{a, b, 0}$ is not cospectral with $H=D_{a^{\prime}, b^{\prime},-1} \cup C_{\delta}$.

Proof. We will consider three cases depending on $\delta$ and $k$. Recall that $a^{\prime} \leqslant b^{\prime}$ and $a^{\prime}+b^{\prime}+\delta=a+b+1$.

Case 1: $\delta<a$

It is easy to see that if $3 \leqslant \delta \leqslant 8$, then the lowest exponent monomial for $D_{1}(a, b, 0 ; x)$ has a negative coefficient, while the lowest exponent monomial for $D_{2}\left(a^{\prime}, b^{\prime},-1 ; x\right)$ (which comes from $\left.x^{6}+2 x^{a^{\prime}}+2 x^{b^{\prime}}\right)$ has a positive coefficient. If $\delta>8$, then $x^{8}$ is the lowest exponent monomial for $D_{1}(a, b, 0 ; x)$, while for $D_{2}\left(a^{\prime}, b^{\prime}-1 ; x\right)$ it is $r x^{t}$ with $t \leqslant 6$.

Case 2: $\delta=a$ and $k=1$

It is easy to observe that for $a \geqslant 7$ the two polynomials are different, indeed in $D_{1}(a, a, 0)$ we have that the minimum exponent is greater than or equal to 7 , while in $D_{2}\left(a^{\prime}, b^{\prime},-1\right)$ the minimum exponent is less than or equal to 6 . If $a=6$, then the coefficient related to $x^{6}$ in $D_{1}(a, a, 0)$ is 2 , while in $D_{2}\left(a^{\prime}, b^{\prime},-1\right)$ the coefficient related to $x^{6}$ is either 1 , or 3 or 5 . If $3 \leqslant a \leqslant 5$, then $a^{\prime}=a<b^{\prime}$. Since $a^{\prime}+b^{\prime}=a+1$, we obtain that $b^{\prime}=1$, impossible.

Case 3: $\delta=a$ and $k \geqslant 2$

The lowest exponent monomial for $D_{1}(a, k a, 0 ; x)$ is either $x^{8}$ (when $a>6$ ) or $3 x^{8}$ (when $a=6$ ) or $2 x^{a+2}$ (when $a<6$ ), while for $D_{2}\left(a^{\prime}, b^{\prime},-1 ; x\right)$ the lowest exponent monomial is either $x^{6}$ (for $a^{\prime}>6$ ) or $r x^{a^{\prime}}$ (for $a^{\prime} \leqslant 6$ ), with $r=2,3,4,5$. 
If $a \geqslant 5$, clearly the two polynomials are different and this implies that $D_{a, k a, 0}$ can not be cospectral with $D_{a^{\prime}, b^{\prime},-1} \cup C_{a}$.

Assume $a=4$, then the lowest exponent monomial for $D_{1}(4,4 k, 0 ; x)$ is $2 x^{6}$, but for $D_{2}\left(a^{\prime}, b^{\prime},-1 ; x\right)$ we have either $x^{6}$ (when $a^{\prime}>6$ ) or $3 x^{6}$ (when $a^{\prime}=6<b^{\prime}$ ) or $5 x^{6}$ (when $\left.a^{\prime}=b^{\prime}=6\right)$, a contradiction.

Finally assume $a=3$, then the lowest exponent monomial for $D_{1}(3,3 k, 0 ; x)$ is $2 x^{5}$. Then it must be $a^{\prime}=5$ and $a^{\prime}<b^{\prime}$. Consequently from $a^{\prime}+b^{\prime}=5+b^{\prime}=3 k+1$ we have $b^{\prime}=3 k-4$ (so $k \geqslant 4$, otherwise $b^{\prime} \leqslant a^{\prime}$ ). If so, by comparing $D_{1}(3,3 k, 0)$ and $D_{2}(5,3 k-4,-1 ; x)$, we have that the two polynomials are different for any $k \geqslant 4$.

This completes the proof.

Now we will compare the lower exponent monomials of $D_{1}(a, b, 0 ; x)$ and $T\left(a_{1}, b_{1}, c_{1} ; x\right)$. In Lemma 4.2 we will consider that $\delta<a$, while in Lemma 4.3 we will consider that $\delta=a$ and, consequently, $b=k a$ for some $k$.

Lemma 4.2. For $\delta<a, D_{a, b, 0}$ is not cospectral with $H=\theta_{a_{1}, b_{1}, c_{1}} \cup C_{\delta}$.

Proof. If $3 \leqslant \delta \leqslant 8$, then the lowest exponent monomial in $D_{1}(a, b, 0 ; x)$ has a negative coefficient, while in $T\left(a_{1}, b_{1}, c_{1} ; x\right)$ it has a positive coefficient.

If $\delta>8$ then the lowest exponent monomial in $D_{1}(a, b, 0 ; x)$ is $x^{8}$, then in $T\left(a_{1}, b_{1}, c_{1} ; x\right)$ it must be $x^{2 a_{1}+6}$ with $a_{1}=1<b_{1}$. If we look to the second lowest exponent monomial in $D_{1}(a, b, 0 ; x)$, i.e. $-2 x^{\delta}$, we have that it has a negative coefficient while in $T\left(a_{1}, b_{1}, c_{1} ; x\right)$ it is positive.

Lemma 4.3. Let $k \geqslant 1$ be an integer. Then

i) for $k=1$ or $k=2, D_{a, k a, 0}$ is determined by the spectrum;

ii) for $k \geqslant 3$, if $D_{a, k a, 0}$ is cospectral with $H=\theta_{a_{1}, b_{1}, c_{1}} \cup C_{a}$ then $a_{1}=1, b_{1}=a-1$ and $c_{1}=(k-1) a-1$.

Proof. The proof is based on two cases according to $k$. Recall that $a_{1} \leqslant b_{1} \leqslant c_{1}$ and $a_{1}+b_{1}+c_{1}=k a-1$.

Case 1: $k=1$

If $a>8$, then $x^{8}$ is the lowest exponent monomial in $D_{1}(a, a, 0)$. The lowest exponent monomial of $T\left(a_{1}, b_{1}, c_{1} ; x\right)$ must be $x^{2 a_{1}+6}$. So we have $a_{1}=1$ and $b_{1}>a_{1}$. If we look to the second lowest exponent monomial we have for $D_{1}(a, a, 0 ; x)$ that it is $2 x^{a}$. If we assume that $2 b_{1}+6=2 c_{1}+6=a$ we get that $a_{1}+b_{1}+c_{1}=a-5 \neq a-1$, a contradiction. So it must be $a_{1}+b_{1}+2=a$, and $b_{1}=a-3$. The latter is a contradiction since $c_{1}=1<b_{1}$.

If $a=8$, then $3 x^{8}$ is the lowest exponent monomial in $D_{1}(a, a, 0)$. It is easy to check that $2 a_{1}+6=2 b_{1}+6=2 c_{1}+6=8$ leads to a contradiction. So it must be $2 a_{1}+6=a_{1}+b_{1}+2=8$, which implies $a_{1}=1$ and $b_{1}=5$, then $c_{1}=1$, a contradiction.

Considering (8), if $a=7$, from $a_{1}+b_{1}+2=7,2 a_{1}+6=8, a_{1}+b_{1}+c_{1}=6$, we obtain $a_{1}=1, b_{1}=4$ and $c_{1}=1$, that is a contradiction. 
Consider $a=6$, then the lowest exponent monomial in $D_{1}(a, a, 0)$ is $2 x^{6}$. So we have that either $2 a_{1}+6=2 b_{1}+6=6$ or $a_{1}+b_{1}+2=6$ with $c_{1}>b_{1}$. From the former we get $a_{1}=b_{1}=0$, impossible (it is a multigraph), from the latter, by considering that $a_{1}+b_{1}+c_{1}=5$, we get a contradiction. The cases $a=3,4,5$ can be done similarly.

Case 2: $k \geqslant 2$

Take $a>6$. Then the lowest exponent monomial for $D_{1}(a, k a, 0 ; x)$ is $x^{8}$. The lowest exponent monomial of $T\left(a_{1}, b_{1}, c_{1} ; x\right)$ must be $x^{2 a_{1}+6}$. So we have $a_{1}=1$ and $b_{1}>a_{1}$. If we look to the second lowest exponent monomial we have for $D_{1}(a, k a, 0 ; x)$ that it is $2 x^{a+2}$. Suppose first that $2 b_{1}+6=2 c_{1}+6=a+2$, since $a_{1}+b_{1}+c_{1}=k a-1$, we get $(k-1) a=-2$, impossible. So the unique possibility is that $a+2=a_{1}+b_{1}+2$ and it must be $b_{1}=a-1$ and $c_{1}>b_{1}$. Since $a_{1}+b_{1}+c_{1}=k a-1, a_{1}=1$ and $b_{1}=a-1$, we get $c_{1}=(k-1) a-1$. Clearly we have that $k \neq 2$ (otherwise $c_{1}=b_{1}$ and the second lowest exponent monomial is different from $\left.2 x^{a+2}\right)$.

Take $a=6$. Then the lowest exponent monomial for $D_{1}(6,6 k, 0 ; x)$ is $3 x^{8}$. Similarly to above we get that $a_{1}=1, b_{1}=5=a-1$ and $c_{1}>b_{1}$. So again it is $c_{1}=(k-1) a-1$, with $k \neq 2$.

Take $a=5$. Then the lowest exponent monomial for $D_{1}(5,5 k, 0 ; x)$ is $2 x^{7}$. Similarly to above we get that it must be $a_{1}+b_{1}+2=7, c_{1}>b_{1}$. The second lowest exponent monomial for $D_{1}(5,5 k, 0 ; x)$ is $x^{8}$ so it must be $a_{1}=1$ and $b_{1}>a_{1}$. So we again get $b_{1}=4=a-1$ and $c_{1}=(k-1) 5-1=(k-1) a-1$, with $k \neq 2$.

Take $a=4$. Then the lowest exponent monomial for $D_{1}(4,4 k, 0 ; x)$ is $2 x^{6}$. Suppose that $2 a_{1}+6=2 b_{1}+6=6$, then we get $a_{1}=b_{1}=0$, impossible (it is a multigraph). So it must be $a_{1}+b_{1}+2=6$ and $b_{1}<c_{1}$. If $k=2$, then $c_{1}=3>b_{1}$ and the unique possibility is that $H=\theta_{2,2,3} \cup C_{4}$, but this graph is not cospectral with $D_{4,8,0}$. So $k \neq 2$. If $k \geqslant 3$, then the second lowest exponent monomial is $-3 x^{8}$. From the latter we have that the unique possibility (note the coefficient is odd) is that $2 a_{1}+6=a_{1}+b_{1}+4=8$, and consequently $a_{1}=1, b_{1}=3=a-1$ and $c_{1}=(k-1) 4-1=(k-1) a-1$.

Finally take $a=3$. Then the lowest exponent monomial for $D_{1}(3,3 k, 0 ; x)$ is $2 x^{5}$. The unique possibility is that $a_{1}+b_{1}+2=5$, so $a_{1}+b_{1}=3$. If $k=2$, then $c_{1}=2>b_{1}$, impossible. So $k \neq 2$. If $k \geqslant 3$, the second lowest exponent monomial is $-4 x^{7}$ which comes from $-4 x^{a_{1}+b_{1}+4}$ (cf. Formula (11)). The third lowest exponent monomial is $3 x^{8}$. Similarly to above we get that $2 a_{1}+6=8$ (in order to get an odd coefficient) and the unique possibility is $a_{1}=1, b_{1}=2$ and $c_{1}=3(k-1)-1$.

The following lemma establishes which are the unique graphs for $a \neq 4$ cospectral with $D_{a, k a, 0}$ (note, the result holds also for $a=4$ ). See also Fig 2.

Lemma 4.4. The graph $D_{a, k a, 0}(k \geqslant 3)$ is cospectral with $H=\theta_{1, a-1,(k-1) a} \cup C_{a}$ if and only if $k=3$.

Proof. We will directly compare their characteristic polynomials through the Schwenk's formulas.

$$
\begin{aligned}
\phi\left(D_{a, k a, 0}\right) & =\phi\left(C_{a}\right) \phi\left(C_{k a}\right)-\phi\left(C_{a}\right) \phi\left(P_{k a-1}\right)-\phi\left(C_{k a}\right) \phi\left(P_{a-1}\right) ; \\
\phi\left(C_{a}\right) \cdot \phi\left(\theta_{1, a-1,(k-1) a-1}\right) & =\phi\left(C_{a}\right)\left[\lambda \phi\left(C_{k a}\right)-2 \phi\left(P_{k a-1}\right)-2 \phi\left(P_{(k-1) a-1}\right)-2 \phi\left(P_{a-1}\right)\right]
\end{aligned}
$$


If we substitute $\lambda=\frac{x^{2}+1}{x}, \phi\left(P_{m}, \lambda\right)=\frac{x^{2 m+2}-1}{x^{m+2}-x^{m}}$ and $\phi\left(C_{m}\right)=x^{m}+x^{-m}-2$, and by equating the above polynomials we get that:

$$
x^{a}\left(x^{2 k+1}-x^{2 k}-x^{k+4}+x^{k+3}-x^{k+1}+x^{k}+x^{4}-x^{3}\right)=0
$$

If $D_{a, k a, 0}$ and $H=\theta_{1, a-1,(k-1) a} \cup C_{a}$ are cospectral then the above polynomial must reduce to the zero polynomial. It is easy to check that (12) is zero for any $x$ if and only if $k=3$. This completes the proof.

By collecting the above results we finally get our main result:

Theorem 4.5. The dumbbell graph $D_{a, b, 0}$ (without cycle $\left.C_{4}\right)$ with $\delta=\operatorname{gcd}(a, b) \geqslant 3$ and $\delta \neq a$ is DAS. For $\delta=a$ (then $b=k a)$, the graph $D_{a, k a, 0}(a \neq 4)$ is DAS if and only if $k \neq 3$. For $k=3$, the graph $D_{a, 3 a, 0}(a \neq 4)$ is $A$-cospectral only with $\theta_{1, a-1,2 a-1} \cup C_{a}$.
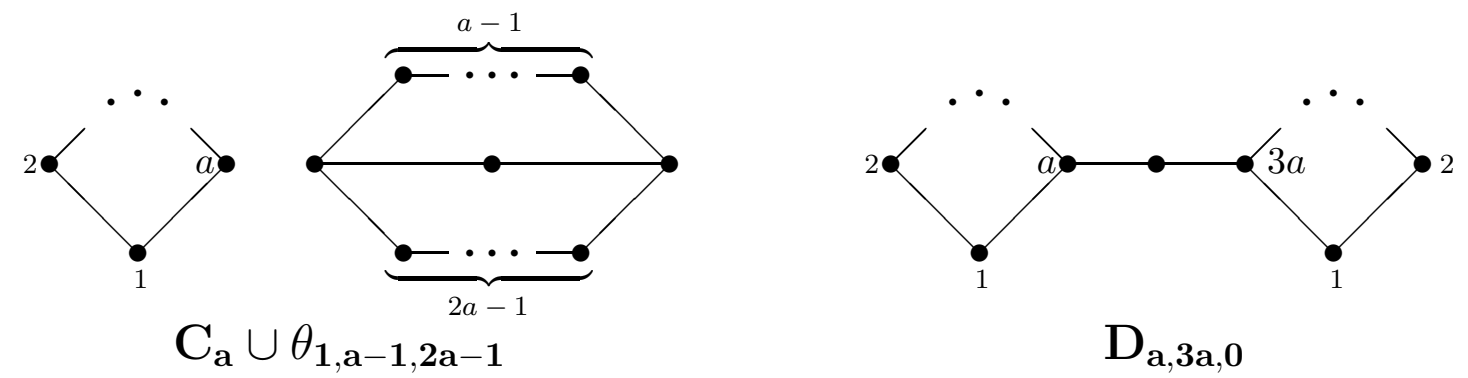

Fig. 2: The $A$-cospectral graphs described in Lemma 4.4 .

By combining the above result and the main result from [12], we obtain:

Theorem 4.6. All dumbbell graphs $D_{a, b, c}$, without cycle $C_{4}$, different from $D_{a, 3 a, 0}$ are determined by the spectrum of the adjacency matrix.

Remark 2. In [12] we proved that most dumbbell graphs are DAS, but it was left to consider dumbbell graphs $D_{a, b, 0}$ with $3 \leqslant \operatorname{gcd}(a, b) \leqslant a$ and $D_{a, b, c}$ with a or $b$ equal to 4 . There is a question: are all dumbbell graphs DAS? Now we have from Theorem 4.5 that the answer is negative. However it remains to consider the case when $D_{a, b, c}$ contains cycle $C_{4}$, i.e. $a$ or $b$ are equal to 4. Note that $D_{4,12,0}$ is $A$-cospectral with $\theta_{1,3,7} \cup C_{4}$ but the latter graph could possibly be not the unique $A$-cospectral graph with $D_{4,12,0}$.

Remark 3. In [9] the authors proved that all dumbbell graphs not containing cycle $C_{4}$ are DAS, clearly their result is not correct since in this paper we detected an exception for $D_{a, 3 a, 0}$. Furthermore the authors of [9] proved that all $\infty$-graphs (denoted in their paper by $b(r, s)$ ) not containing $C_{4}$ are $D A S$, but we got an exception for $b(2 r, 2 r+2)$ (cf. Proof of Lemma 6.12 in [13]). 


\section{$5 \quad Q$-spectral characterization of dumbbell graphs}

In [13] we showed that from the $A$-spectral characterization of a graph, we can deduce its $Q$-spectral characterization. Since in our papers we got that, for $a \geqslant 6$ all dumbbell graphs $D_{a, b, c}$ with $c \neq 0$ or $c=0$ and $b \neq 3 a$, are DAS, then we are able to easily extend such results to the $Q$-theory of graph spectra.

The following results can be found in [13] (cf. also [3, 4]). However in order to make this paper self-contained we report them here. Recall, we will say that two graphs $G$ and $H$ are $A$-cospectral if and only if their $A$-spectra are the same. Similarly, $G$ and $H$ are $Q$ cospectral if and only if their $Q$-spectra are the same. A graph $G$ that is determined by the adjacency (signless Laplacian) spectrum will said to be a DAS (resp. DQS) graph. $\varphi(G)$ denotes the $Q$-characteristic polynomial of $G$, while $\phi(G)$ denotes the $A$-characteristic polynomial of $A(G)$. Finally if $G$ is a graph, then $S(G)$ denotes the subdivision graph of $G$, obtained from $G$ by inserting a vertex of degree 2 in each edge of $G$.

The following lemma can be found in many references, see [3, 14] for example.

Lemma 5.1. Let $G$ be a graph of order $n$ and size $m$, and $S(G)$ be the subdivision graph of $G$. Then

$$
\phi(S(G), \lambda)=\lambda^{m-n} \varphi\left(G, \lambda^{2}\right) .
$$

Theorem 5.2. Let $G$ be a graph of order $n$ and size $m$, and $S(G)$ be the subdivision graph of $G$.

(i) Graphs $G$ and $H$ are $Q$-cospectral if and only if $S(G)$ and $S(H)$ are $A$-cospectral;

(ii) Let $G$ be a graph and $S(G)$ a DAS-graph. Then $G$ is a DQS-graph;

(iii) Let $G$ be a DQS-graph. If any graph A-cospectral with $S(G)$ is a subdividion of some graph, then $S(G)$ is a DAS-graph.

Proof. (i) Since $G$ and $H$ are $Q$-cospectral, then $\varphi(G, \lambda)=\varphi(H, \lambda)$. By Lemma 2.4, $G$ and $H$ have the same order and size, which implies that $m(G)-n(G)=m(H)-n(H)$. Thus,

$$
\lambda^{m(G)-n(G)} \varphi\left(G, \lambda^{2}\right)=\lambda^{m(H)-n(H)} \varphi\left(H, \lambda^{2}\right),
$$

which shows by Lemma 5.1 that $\phi(S(G), \lambda)=\phi(S(H), \lambda)$. This ends the necessity.

Conversely, assume that $S(G)$ and $S(H)$ are $A$-cospectral, then

$$
\phi(S(G), \lambda)=\phi(S(H), \lambda), \quad n(S(G))=n(S(H)), \quad m(S(G))=m(S(H)) .
$$

Note that for any graph $G$ we have

$$
m(S(G))=2 m(G), \quad n(S(G))=m(G)+n(G) .
$$

Hence, from $n(S(G))=n(S(H))$ and $m(S(G))=m(S(H))$, we obtain that $m(G)=m(H)$ and $n(G)=n(H)$. So we get that

$$
(\sqrt{\lambda})^{n(G)-m(G)} \phi(S(G), \sqrt{\lambda})=(\sqrt{\lambda})^{n(H)-m(H)} \phi(S(G), \sqrt{\lambda}),
$$


which shows from Lemma 5.1 that $\varphi(G, \lambda)=\varphi(H, \lambda)$.

(ii) Set $\varphi(H, \lambda)=\varphi(G, \lambda)$. Then by (i) we get $\phi(S(H), \lambda)=\phi(S(G), \lambda)$. Since $S(G)$ is a DAS-graph, then $S(H) \cong S(G)$ which shows that $H \cong G$.

(iii) Without loss of generality, let $H$ and $H^{\prime}$ be two graphs such that $H=S\left(H^{\prime}\right)$ and $\phi(H, \lambda)=\phi\left(S\left(H^{\prime}\right), \lambda\right)=\phi(S(G), \lambda)$, which implies from (i) that $\varphi\left(H^{\prime}, \lambda\right)=\varphi(G, \lambda)$. Since $G$ is a DQS-graph, then $H^{\prime} \cong G$, and so $H=S\left(H^{\prime}\right) \cong S(G)$ which shows that $S(G)$ is a DAS-graph.

Since the subdivision of a dumbbell graph $D_{a, b, c}$ is the dumbbell graph $D_{2 a, 2 b, 2(c+1)}$, by combining Theorems 4.6 and 5.2 , we are able to state the following theorem:

Theorem 5.3. All dumbbell graphs $D_{a, b, c}$ different from $D_{a, 3 a,-1}$, are determined by the spectrum of the signless Laplacian matrix. The graph $D_{a, 3 a,-1}$ is $Q$-cospectral only with $\theta_{0, a-1,2 a-1} \cup C_{a}$.

Proof. Any dumbbell graph $D_{a, b, c}$ with $c \geqslant 0$ is surely DQS. Indeed $S\left(D_{a, b, c}\right)=D_{2 a, 2 b, 2(c+1)}$ and from $a \geqslant 3$ and $c \geqslant 0$ we have that, by Theorem 4.6, $D_{2 a, 2 b, 2(c+1)}$ is DAS. Hence by Theorem 5.2, $D_{a, b, c}(c \neq-1)$ is determined by the signless Laplacian spectrum.

Take $c=-1$, then $S\left(D_{a, b,-1}\right)=D_{2 a, 2 b, 0}$. By Theorem 4.6 we have that if $2 b \neq 3(2 a)$, hence $b \neq 3 a$, then, similarly to above, we get that also $D_{a, b,-1}$ is DQS. Finally, if $c=-1$ and $b=3 a$, then $\phi\left(S\left(D_{a, 3 a,-1}\right)\right)=\phi\left(D_{2 a, 6 a, 0}\right)=\phi\left(\theta_{1,2 a-1,4 a-1}\right) \phi\left(C_{2 a}\right)=\phi\left(S\left(\theta_{0, a-1,2 a-1} \cup\right.\right.$ $C_{a}$ ). The latter implies $\varphi\left(D_{a, 3 a,-1}\right)=\varphi\left(\theta_{0, a-1,2 a-1} \cup C_{a}\right)$ (cf. Fig. 3).

This completes the proof.
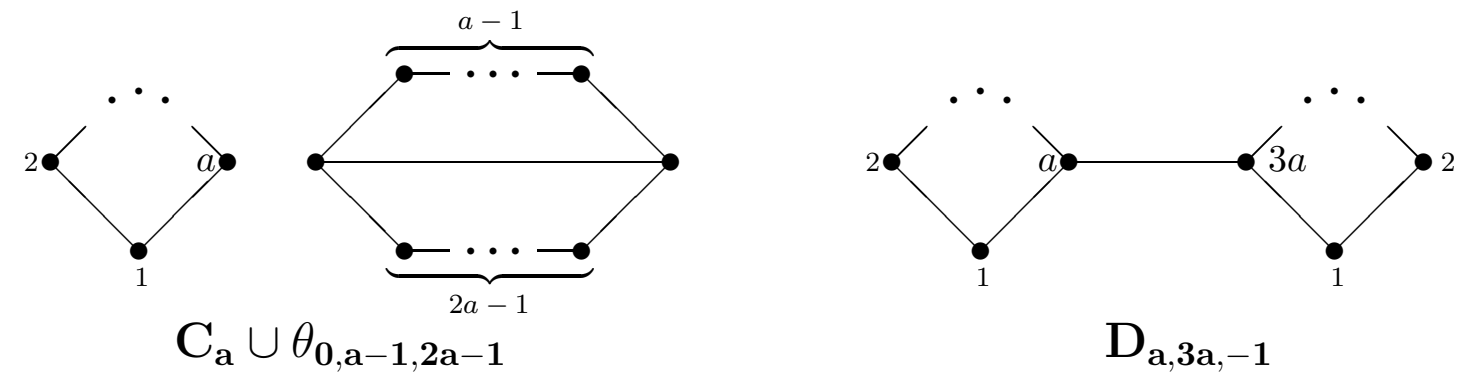

Fig. 3: A pair of non-isomorphic $Q$-cospectral graphs.

Remark 4. Similarly to above, since the subdivision of a $\theta$-graph is still a $\theta$-graph but without cycle $C_{4}$, we have from the main result of [11] that all $\theta$-graphs are DQS-graphs.

\section{Acknowledgement}

The authors express their thanks to the anonymous referee whose comments and suggestions improved the final form of this manuscript. 


\section{References}

[1] R. Boulet, B. Jouve, The lollipop graph is determined by its spectrum, Electron. J. Combin. 15 (2008) \#R74.

[2] D. Cvetković, M. Doob, H. Sachs, Spectra of Graphs - Theory and Applications, III revised and enlarged edition, Johan Ambrosius Bart Verlag, Heidelberg - Leipzig, 1995.

[3] D. Cvetković, P. Rowlinson, S.K. Simić, Eigenvalue bounds for the signless Laplacians, Publ. Inst. Math. (Beograd) (N.S.) 81 (95) (2007) 11-27.

[4] D. Cvetković, S.K. Simić, Towards a spectral theory of Graphs based on signless Laplacian, II, Linear Algebra Appl. 432 (2010) 2257-2272.

[5] D. Cvetković, S.K. Simić, Towards a spectral theory of Graphs based on signless Laplacian, III, Appl. Anal. Discrete Math. (2010), doi:10.2298/AADM1000001C, in press.

[6] E.R. van Dam, W.H. Haemers, Which graphs are determined by their spectra?, Linear Algebra Appl. 373 (2003) 241-272.

[7] E.R. van Dam, W.H. Haemers, Developments on spectral characterizations of graphs, Discrete Math. 309 (2009) 576-586.

[8] N. Ghareghani, G.R. Omidi, B. Tayfeh-Rezaie, Spectral characterization of graphs with index at most $\sqrt{2+\sqrt{5}}$, Linear Algebra Appl. 420 (2007) 483-489.

[9] X. Fan, Y. Luo, Spectral characterization of dumbbell graphs, Linear Algebra Appl. (2009), doi: 10.1016/j.laa.2009.11.012, in press.

[10] W.H. Haemers, X.G. Liu, Y.P. Zhang, Spectral characterizations of lollipop graphs, Linear Algebra Appl. 428 (2008) 2415-2423.

[11] F. Ramezani, N. Broojerdian, B. Tayfeh-Rezaie, A note on the spectral characterization of $\theta$-graphs, Linear Algebra Appl. 431 (2009) 626-632.

[12] J.F. Wang, Q.X. Huang, F. Belardo, E.M. Li Marzi, A note on the spectral characterization of dumbbell graphs, Linear Algebra Appl. 431 (2009) 1707-1714.

[13] J.F. Wang, Q.X. Huang, F. Belardo, E.M. Li Marzi, On the spectral characterizations of $\infty$-graphs, Discrete Math. (2010), doi:10.1016/j.disc.2010.01.021, in press.

[14] J.F. Wang, Q.X. Huang, F. Belardo, E.M. Li Marzi, On graphs whose signless Laplacian index does not exceed 4.5, Linear Algebra Appl. 431 (2009) 162-178. 